\title{
Superiority of entangled measurements over all local strategies for the estimation of product coherent states
}

Nisset, J.; Acin, A.; Andersen, Ulrik Lund; Cerf, N.J.; García-Patrón, R.; Navascues, M.; Sabuncu, M.

Published in:

Physical Review Letters

Link to article, DOI:

10.1103/PhysRevLett.98.260404

Publication date:

2007

Document Version

Publisher's PDF, also known as Version of record

Link back to DTU Orbit

Citation (APA):

Nisset, J., Acin, A., Andersen, U. L., Cerf, N. J., García-Patrón, R., Navascues, M., \& Sabuncu, M. (2007).

Superiority of entangled measurements over all local strategies for the estimation of product coherent states.

Physical Review Letters, 98(26), 260404. https://doi.org/10.1103/PhysRevLett.98.260404

\section{General rights}

Copyright and moral rights for the publications made accessible in the public portal are retained by the authors and/or other copyright owners and it is a condition of accessing publications that users recognise and abide by the legal requirements associated with these rights.

- Users may download and print one copy of any publication from the public portal for the purpose of private study or research.

- You may not further distribute the material or use it for any profit-making activity or commercial gain

- You may freely distribute the URL identifying the publication in the public portal 


\title{
Superiority of Entangled Measurements over All Local Strategies for the Estimation of Product Coherent States
}

\author{
J. Niset, ${ }^{1}$ A. Acín, ${ }^{2}$ U. L. Andersen, ${ }^{3,4}$ N. J. Cerf, ${ }^{1}$ R. García-Patrón, ${ }^{1}$ M. Navascués, ${ }^{2}$ and M. Sabuncu ${ }^{3}$ \\ ${ }^{1}$ Quantum Information and Communication, Ecole Polytechnique, CP 165, Université Libre de Bruxelles, 1050 Brussels, Belgium \\ ${ }^{2}$ ICFO-Institut de Ciencies Fotoniques, Mediterranean Technology Park, 08860 Castelldefels (Barcelona), Spain \\ ${ }^{3}$ Institut für Optik, Information und Photonik, Max-Planck Forschungsgruppe, Universität Erlangen-Nürnberg, \\ Günther-Scharowsky str. 1, 91058, Erlangen, Germany \\ ${ }^{4}$ Department of Physics, Technical University of Denmark, Building 309, 2800 Kgs. Lyngby, Denmark
}

(Received 29 August 2006; published 26 June 2007)

It is shown that the ensemble $\left\{P(\alpha),|\alpha\rangle\left|\alpha^{*}\right\rangle\right\}$, where $P(\alpha)$ is a Gaussian distribution of finite variance and $|\alpha\rangle$ is a coherent state, can be better discriminated with an entangled measurement than with any local strategy supplemented by classical communication. Although this ensemble consists of products of quasiclassical states without any squeezing, it thus exhibits a purely quantum feature. This remarkable effect is demonstrated experimentally by implementing the optimal local strategy on coherent states of light together with a global strategy that yields a higher fidelity.

DOI: 10.1103/PhysRevLett.98.260404

Entanglement is known to be a valuable resource, which can be used to achieve a large variety of quantum protocols, mostly through the manipulation and detection of entangled states. Because of the nonlocal nature of entanglement, joint measurements are typically necessary to properly access the information stored in the quantum correlations of entangled states. But entanglement has proven to be much richer than that. It can, for example, take profit of purely classical correlations to provide a better access to the information encoded in a product state [1]. In particular, the optimal measurement of $N$ identically prepared qubits is known to be a joint entangled measurement [2]. Moreover, some classical correlations perform better than others: a surprising result, obtained in [3], is that more information can be extracted from a pair of orthogonal qubits than from two identical qubits. An even more intriguing phenomenon, named "nonlocality without entanglement" [4], is the existence of ensembles of orthogonal product states that cannot be perfectly distinguished using local operations and classical communications (LOCC) only, but can be perfectly discriminated through a joint-yet separable — measurement.

In the recent years, much attention has been devoted to quantum information based on continuous variables (CV). Many results initially derived for qubits have been successfully adapted to infinite dimensional systems. It is thus tempting to ask whether similar quantum effects may be observed with $\mathrm{CV}$ product states. In this Letter, we answer this question affirmatively by exhibiting an ensemble of classically-correlated product coherent states that can be better discriminated when the parties act globally rather than locally. Then, we report on the experimental demonstration of this surprising property based on the sideband encoding of a modulated laser beam with no need for squeezing. The superior discrimination of product states via entangled measurements was verified experimentally only very recently for two qubits $[5,6]$. To our knowledge,
PACS numbers: 03.65.Ud, 03.67.Mn, 42.50.Ct, 42.50.Xa

the present work achieves the first experimental evidence of such a quantum effect with continuous variables.

Here, we consider the ensemble of bipartite product states $\left\{P(\alpha),|\alpha\rangle\left|\alpha^{*}\right\rangle\right\}$, with $P(\alpha)$ being a Gaussian distribution and $|\alpha\rangle$ a coherent state, which was introduced in the context of the optimal phase-conjugation transformation [7]. We show that it can be better discriminated having access to joint operations than being restricted to LOCC. In this respect, we have an ensemble of quasiclassical states which are neither squeezed, nor entangled, but nevertheless exhibit some peculiar nonclassical property. This may be viewed as a nonlocal effect without squeezing, though nonlocality should not be understood here as the incompatibility with local-hidden-variable models (unlike the case of Bell tests), but rather as the manifestation of an inherently global property.

To estimate the quality of a particular measurement strategy we will use the mean fidelity, i.e.,

$$
F=\sup _{M_{y}} \sup _{\rho_{y}} \sum_{y} \int d \alpha P(\alpha)\left\langle\alpha\left|\left\langle\alpha^{*}\left|M_{y}\right| \alpha\right\rangle\right| \alpha^{*}\right\rangle\left\langle\alpha\left|\rho_{y}\right| \alpha\right\rangle
$$

where $M_{y}$ are the positive operators defining the measurement, $\sum_{y} M_{y}=1$, and $\rho_{y}$ are states prepared according to the measurement results. This means that our goal is to optimally measure $|\alpha\rangle\left|\alpha^{*}\right\rangle$ and prepare a state as close as possible to $|\alpha\rangle$. To bound the fidelity achievable by local operations, we will first show that the optimal LOCC strategies on $|\alpha\rangle\left|\alpha^{*}\right\rangle$ and $|\alpha\rangle|\alpha\rangle$ yield equal fidelities. We next prove that the optimal measure-and-prepare strategy on identical copies of $|\alpha\rangle$ is achieved by a local strategy. And finally, we exhibit a joint measurement on phaseconjugate states, conjectured to be optimal, which gives a higher fidelity than the optimal strategy on two identical copies. We will thus prove that

$$
F_{L}^{*}=F_{L}=F<F^{*},
$$


where $F$ and $F^{*}\left(F_{L}\right.$ and $\left.F_{L}^{*}\right)$ denote the optimal fidelities for global (local) measurements on $|\alpha\rangle|\alpha\rangle$ and $|\alpha\rangle\left|\alpha^{*}\right\rangle$ respectively.

Let us start by proving that local strategies on $|\alpha\rangle\left|\alpha^{*}\right\rangle$ and $|\alpha\rangle|\alpha\rangle$ give identical fidelities, i.e., $F_{L}^{*}=F_{L}$. Recall that any LOCC strategy consists of a sequence of correlated measurements plus a decision strategy depending on the observed statistics. After $n$ rounds of measurements, the relevant probabilities can be written as

$$
\operatorname{Pr}(\beta)=\operatorname{Tr}\left\{\left(A_{\beta} \otimes B_{\beta}\right)|\alpha\rangle\left\langle\alpha|\otimes| \alpha^{*}\right\rangle\left\langle\alpha^{*}\right|\right\}
$$

with the positive operators $A_{\beta}$ and $B_{\beta}$ defined as

$$
\begin{aligned}
A_{\beta} & =A_{r_{n}}^{n}\left(r_{1}, r_{2}, \ldots, r_{n-1}\right) \ldots A_{r_{3}}^{3}\left(r_{1}, r_{2}\right) A_{r_{1}}^{1} \\
B_{\beta} & =B_{r_{n-1}}^{n-1}\left(r_{1}, r_{2}, \ldots, r_{n-2}\right) \ldots B_{r_{2}}^{2}\left(r_{1}\right) .
\end{aligned}
$$

In this expression, $r_{i}$ is the outcome of the $i$ th measurement, and the upper index stands for its order in the sequence of measurements. These operators depend on the decision strategy, and are constrained by the measurement normalization conditions. Now, suppose that a particular LOCC strategy is optimal for $|\alpha\rangle\left|\alpha^{*}\right\rangle$ and gives the fidelity $F_{L}^{*}$. We can easily map this optimal strategy into an optimal LOCC strategy for $|\alpha\rangle|\alpha\rangle$. Indeed, replacing $B_{\beta}$ by $B_{\beta}^{*}$, one defines another LOCC sequence of measurements that achieves the same fidelity for $|\alpha\rangle|\alpha\rangle$ since the trace of Hermitian operators is invariant under complex conjugation, hence $F_{L}^{*}=F_{L}$.

Next, let us prove that the optimal measure-and-prepare strategy on $|\alpha\rangle|\alpha\rangle$ is a local strategy, i.e., $F_{L}=F$. Note that this result is already known for a distribution of infinite width [8], using the variances of the estimated quadratures as a figure of merit. Here, we prove a more powerful result: we consider the realistic case of finite-width distributions, and do not make any assumption on the measurement nor the reconstruction. Actually, we prove the more general result that the optimal strategy for $N$ copies of a coherent state distributed according to a Gaussian of variance $1 / \lambda$ yields a fidelity $F^{N}$ satisfying

$$
F^{N} \leq \frac{N+\lambda}{N+\lambda+1} .
$$

This upper bound is exactly the fidelity achieved by $N$ independent heterodyne measurements, followed by the preparation of a coherent state centered on $\frac{1}{N+\lambda} \sum_{i=1}^{N} \alpha_{i}$ (with $\alpha_{i}$ the result of the $i$ th measurement).

Without loss of generality, we can restrict our optimization to measurements consisting of projectors $\left|\Phi_{y}\right\rangle\left\langle\Phi_{y}\right|$ and preparation of pure states $\left|\chi_{y}\right\rangle$. For the input states $|\alpha\rangle^{\otimes N}$ distributed with the Gaussian distribution $P(\alpha)=$ $\frac{\lambda}{\pi} \exp \left(-\lambda|\alpha|^{2}\right)$, the average fidelity reads

$$
F=\sum_{y} \int d \alpha P(\alpha)\left|\left\langle\alpha^{\otimes N} \mid \Phi_{y}\right\rangle\right|^{2}\left|\left\langle\alpha \mid \chi_{y}\right\rangle\right|^{2} .
$$

To bound this fidelity, we generalize a method used in [9] to calculate the optimal fidelity for a measure-and-prepare strategy on a single copy of a coherent state $|\alpha\rangle$ distributed according to $P(\alpha)$. First, one needs to realize that we can concentrate the $N$ modes of $|\alpha\rangle^{\otimes N}$ into one single mode $|\sqrt{N} \alpha\rangle$ by means of beam splitters. This operation is unitary and completely reversible; hence, it will not change the fidelity. We can thus write

$$
\begin{aligned}
F & =\sum_{y} \int d \alpha P(\alpha)\left|\left\langle\sqrt{N} \alpha \mid \phi_{y}\right\rangle\right|^{2}\left|\left\langle\alpha \mid \chi_{y}\right\rangle\right|^{2} \\
& \leq \sup _{\phi_{y}, \chi_{y}} \sum_{y}\left\langle\chi_{y}\left|A_{\phi_{y}}\right| \chi_{y}\right\rangle=\sup _{\phi_{y}} \sum_{y}\left\|A_{\phi_{y}}\right\|_{\infty}
\end{aligned}
$$

after introduction of the operators

$$
A_{\phi_{y}}=\int d \alpha P(\alpha)\left|\left\langle\sqrt{N} \alpha \mid \phi_{y}\right\rangle\right|^{2}|\alpha\rangle\langle\alpha| .
$$

The last equality of (4) is trivial, as it is indeed best to prepare the eigenstate of $A_{\phi_{y}}$ associated to the largest eigenvalue for a given outcome $y$. We can now turn to the core of the method. Following [9], we first prove that

$$
\left\|A_{\phi}\right\|_{p} \leq \frac{N+\lambda}{\left[(N+\lambda+1)^{p}-1\right]^{1 / p}}\left\|A_{\phi}\right\|_{1}
$$

holds for all states $|\phi\rangle$ and all $p$ norms $\|A\|_{p}=$ $\left(\operatorname{Tr}\left\{|A|^{p}\right\}\right)^{1 / p}$. The limiting case $p \rightarrow \infty$, in combination with the measurement normalization $\sum_{y}\left|\phi_{y}\right\rangle\left\langle\phi_{y}\right|=1$ (which implies $\sum_{y}\left\|A_{\phi_{y}}\right\|_{1}=1$ ), is then sufficient to prove Eq. (2). We only present here the main results of our calculation. An interested reader should consult $[9,10]$ for more details.

The properties of the trace allow us to write

$$
\begin{aligned}
& \left\|A_{\phi}\right\|_{p}^{p}=\operatorname{Tr}\left\{A_{\phi}^{p}\right\}=\operatorname{Tr}\left\{|\phi\rangle\left\langle\left.\phi\right|^{\otimes p} B\right\},\right. \\
& \left\|A_{\phi}\right\|_{1}^{p}=\operatorname{Tr}\left\{A_{\phi}\right\}^{p}=\operatorname{Tr}\left\{|\phi\rangle\left\langle\left.\phi\right|^{\otimes p} C\right\},\right.
\end{aligned}
$$

where we have defined the operators $B$ and $C$ as

$$
\begin{aligned}
B= & \iint d \alpha_{1} \ldots d \alpha_{p} P\left(\alpha_{1}\right) \ldots P\left(\alpha_{p}\right) \\
& \times\left\langle\alpha_{1} \mid \alpha_{2}\right\rangle \ldots\left\langle\alpha_{p} \mid \alpha_{1}\right\rangle\left|\sqrt{N} \alpha_{1}\right\rangle\left\langle\sqrt{N} \alpha_{1}\right| \otimes \ldots \\
& \otimes\left|\sqrt{N} \alpha_{p}\right\rangle\left\langle\sqrt{N} \alpha_{p}\right|, \\
C= & \bigotimes_{i=1}^{p} \int d \alpha_{i} P\left(\alpha_{i}\right)\left|\sqrt{N} \alpha_{i}\right\rangle\left\langle\sqrt{N} \alpha_{i}\right| .
\end{aligned}
$$

These two operators can be diagonalized in the same basis. A unitary transformation turns them into tensor products of unnormalized thermal states, which are diagonal in the corresponding Fock state basis. Expressing the product state $|\phi\rangle^{\otimes p}$ in this Fock state basis and remembering that $\operatorname{Tr}\left\{|\phi\rangle\left\langle\left.\phi\right|^{\otimes p} B\right\} \geq 0\right.$, one finds that

$$
\operatorname{Tr}\left\{|\phi\rangle\left\langle\left.\phi\right|^{\otimes p} B\right\} \leq \frac{(N+\lambda)^{p}}{(N+\lambda+1)^{p}-1} \operatorname{Tr}\left\{|\phi\rangle\left\langle\left.\phi\right|^{\otimes p} C\right\} .\right.\right.
$$

The $p$ th root of this expression gives directly relation (6) and thus completes the proof of relation (2). 
Now, let us prove the existence of a joint measurement on phase-conjugate states that yields a higher fidelity than (2) for $N=2$. One such measurement was introduced in [7] in order to show that the $|\alpha\rangle\left|\alpha^{*}\right\rangle$ encoding outperforms $|\alpha\rangle|\alpha\rangle$ in the case $\lambda=0$. The strategy is the following. First, the two modes are sent on a beam splitter (BS), which outputs two coherent states displaced along the $x$ and $p$ axis, respectively, i.e., $|\alpha\rangle\left|\alpha^{*}\right\rangle \rightarrow\left|x_{\alpha}, 0\right\rangle\left|0, p_{\alpha}\right\rangle$, where $\alpha=$ $\left(x_{\alpha}+i p_{\alpha}\right) / \sqrt{2}$. Next, the appropriate quadratures are measured on the two output ports, and some state $\left|f_{\beta}\right\rangle$ is reconstructed according to the measurement outcomes.

That this strategy outperforms the optimal measurement on identical copies has an intuitive explanation. Suppose we apply this BS strategy to the $|\alpha\rangle|\alpha\rangle$ case. Then, the two modes are concentrated on one output port, so that only heterodyning can extract information about $x$ and $p$ simultaneously; that is, we need to combine the state with vacuum at another BS, introducing extra noise. Nevertheless, according to the previous section, this strategy is optimal. Applying this same strategy to the $|\alpha\rangle\left|\alpha^{*}\right\rangle$ state, we can directly access the entire information by homodyning each of the two output modes of the BS. Since we do not introduce vacuum in this setup while detecting the same mean signal, we have less noise and can therefore expect a greater fidelity.

In order to calculate this fidelity and easily compare with (2), suppose that we have at our disposal $N$ coherent states made of $N / 2$ pairs $|\alpha\rangle\left|\alpha^{*}\right\rangle$, or equivalently one pair $|\sqrt{N / 2} \alpha\rangle\left|\sqrt{N / 2} \alpha^{*}\right\rangle$. The corresponding fidelity reads

$$
\begin{aligned}
F_{\mathrm{BS}}^{* N} & =\iint P(\alpha) P\left(x, p \mid x_{\alpha}, p_{\alpha}\right)\left|\left\langle f_{\beta} \mid \alpha\right\rangle\right|^{2} d x d p d \alpha \\
& =2 \frac{\lambda}{\pi^{2}} \int e^{-2|\beta|^{2}}\left\langle f_{\beta}\left|\hat{O}_{\beta}\right| f_{\beta}\right\rangle d \beta,
\end{aligned}
$$

where $P\left(x, p \mid x_{\alpha}, p_{\alpha}\right)$ is the probability to measure $(x, p)$ by homodyning on $\left|\sqrt{N / 2} x_{\alpha}\right\rangle\left|\sqrt{N / 2} p_{\alpha}\right\rangle, \hat{O}_{\beta}$ a known semidefinite Hermitian operator, and $\beta=(x+i p) / \sqrt{2}$. Optimization of this fidelity with respect to the reconstructed state boils down to finding the largest eigenvalue of this operator $\hat{O}_{\beta}$. We can calculate this value analytically as in [11], yielding the maximum fidelity

$$
F_{\mathrm{BS}}^{* N}=\frac{2 N+\lambda}{2 N+\lambda+1}
$$

and the corresponding eigenvector $\left|f_{\beta}\right\rangle=\left|\frac{2 \sqrt{N}}{2 N+\lambda} \beta\right\rangle$. Clearly, (10) is larger than (2) for any $N$, so we conclude that $F^{N}<F_{\mathrm{BS}}^{* N} \leq F^{* N}$, where $F^{* N}$ is the optimal fidelity of a global strategy for $N / 2$ phase-conjugated pairs.

Interestingly, $F_{\mathrm{BS}}^{* N}=F^{2 N}$, that is, this global strategy on $|\alpha\rangle\left|\alpha^{*}\right\rangle$ is exactly as efficient as the optimal strategy on $|\alpha\rangle^{\otimes 4}$. Again, this has an intuitive explanation. Consider the input state $|\alpha\rangle^{\otimes 4}$. It can be concentrated using two BS, namely, $|\alpha\rangle^{\otimes 4} \rightarrow|\sqrt{2} \alpha\rangle^{\otimes 2}$. Because dual homodyning on $|\sqrt{2} \alpha\rangle|\sqrt{2} \alpha\rangle$ or $\left|x_{\alpha}\right\rangle\left|p_{\alpha}\right\rangle$ gives identical statistics, the corresponding fidelities are equal.
Now, let us proceed with the experimental demonstration of the quantum feature exhibited by $\left\{|\alpha\rangle\left|\alpha^{*}\right\rangle\right\}$. The laser used in our experiment is a monolithic Nd:YAG laser producing a field at $1064 \mathrm{~nm}$, which is split into two parts and subsequently directed into the coherent state preparation stage (see Fig. 1). To ensure that the information is encoded as pure coherent states, the states are assumed to be residing at a radio frequency sideband defined within a certain bandwidth of the laser beam. In addition to the high degree of purity, the sideband encoding also holds the advantage of allowing for easy low-voltage control of the coherent amplitudes via simple electro-optic modulators operating at the sideband frequency [12]. Note, therefore, that the two beams are bright although the particular sidebands in question are vacuum states, $\{|0\rangle\}$, before the encoding. The production of the two phase-conjugate coherent states, $|\alpha\rangle$ and $\left|\alpha^{*}\right\rangle$, is then performed by displacing the vacuum sidebands using an amplitude modulator (AM) and a phase modulator (PM) in each arm as shown in Fig. 1. The two states are prepared by using the same signal generator, that is by communicating classically correlated information between the two preparation stations. The relative phase shift of $\pi$ between the phase quadratures was established by adjusting the cable lengths appropriately.

First, we characterize the prepared states by measuring the two copies individually, by successive use of a heterodyne detector yielding information about the amplitude and phase quadratures, simultaneously. The coherent state is combined with a phase stabilized auxiliary beam at a 50:50 beam splitter with a $\pi / 2$ relative phase shift and balanced intensities. They interfere with a contrast of $99 \%$ and the two output beams are detected with high quantum efficiency $(95 \%)$ photodiodes. Subsequently, the photocurrents are subtracted and added, which provides information about the phase and amplitude quadratures, respectively. Finally, the spectral densities of the quadratures are recorded on a spectrum analyzer. Using the fact that the heterodyne detector projects the signal under investigation
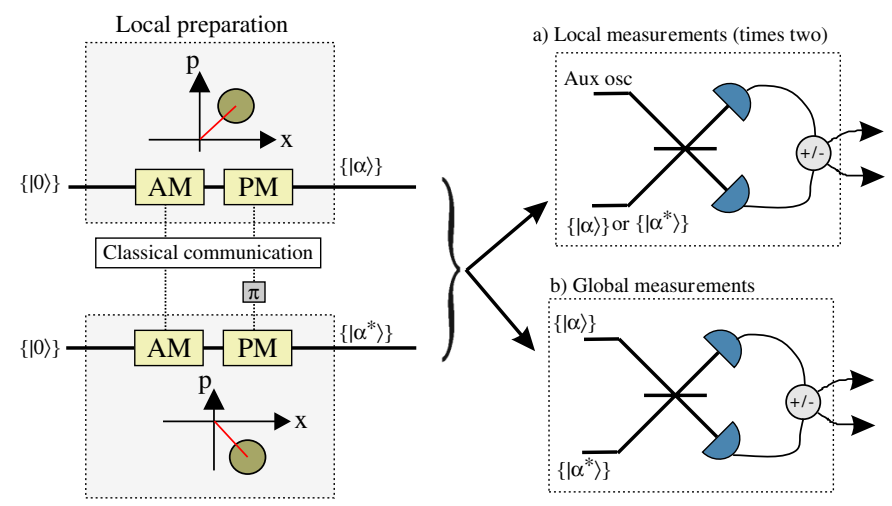

FIG. 1 (color online). Schematic of the experimental setup. The states are measured using (a) a local strategy and (b) a global strategy. 

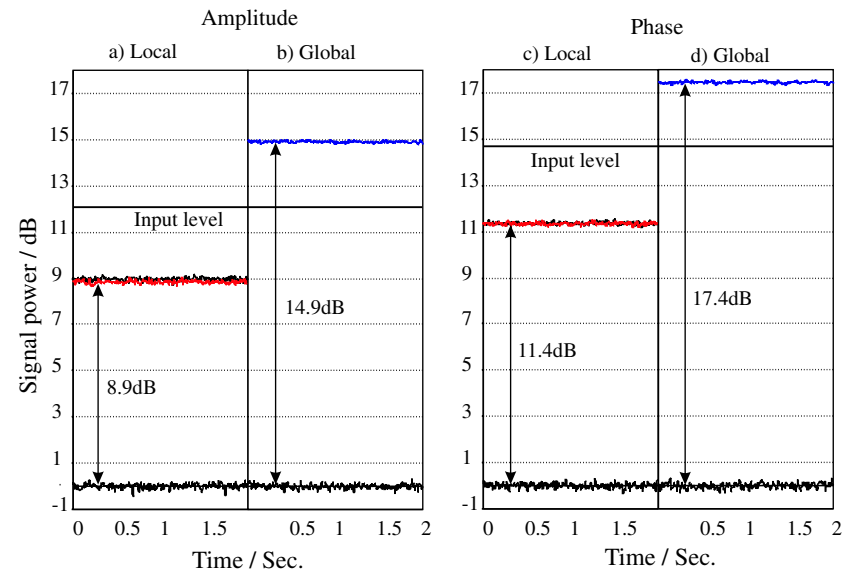

FIG. 2 (color online). Spectral power densities (normalized to the quantum noise level) of the local and global strategies. The resolution bandwidth is $100 \mathrm{kHz}$ and the video bandwidth is $30 \mathrm{~Hz}$.

onto a vacuum state, we easily infer the spectral densities of the prepared copies. Furthermore, the measurements have also been corrected to account for the detection losses and electronic dark noise in order to avoid an erroneous underestimation. The inferred results for the spectral densities are shown by the solid horizontal lines in Fig. 2.

These measurements for characterization of the prepared copies are in fact identical to the measurements associated with an optimal local estimation strategy. However, in contrast to the characterization, for the estimation of unknown coherent states we are not correcting for detector losses and electronic dark noise. The individual spectral densities for local measurements of $|\alpha\rangle$ and $|\alpha *\rangle$ are shown in column (a) and (c) of Fig. 2. From these measurements we find the added noise to be $\Delta_{x}=\Delta_{p}=$ $1.12 \pm 0.04$ for the amplitude and phase quadratures. Assuming a flat distribution of coherent states, the fidelity is given by

$$
F=\frac{2}{\sqrt{\left(2+\Delta_{x}\right)\left(2+\Delta_{p}\right)}}
$$

and calculated to $F_{L}=64.0 \pm 1 \%$. This is close to the theoretical maximum of $2 / 3$.

We now discuss the experimental realization of our joint measurement of the phase-conjugate copies. As mentioned above, this strategy is to combine the two copies at a 50:50 beam splitter and subsequently measure the amplitude quadrature in one output and the phase quadrature in the other output port of the beam splitter. Such a strategy measures the combinations $\hat{x}_{1}+\hat{x}_{2}$ and $\hat{p}_{1}-\hat{p}_{2}$, where the indices refer to the two input modes. This combination can, however, be accessed using an experimentally simpler approach since the information is encoded onto sidebands of two equally intense bright beams (with the power $60 \mu \mathrm{W}$ ). The two classically correlated copies are carefully mode matched $(\sim 99 \%)$ at a 50:50 beam splitter and actively locked to have balanced intensities at the outputs of the beam splitter. Directly measuring the two outputs yields the quadrature combinations $\hat{i}_{1}=\left(\hat{x}_{1}+\hat{x}_{2}+\hat{p}_{1}-\hat{p}_{2}\right) / 2$ and $\hat{i}_{2}=\left(\hat{x}_{1}+\hat{x}_{2}-\hat{p}_{1}+\hat{p}_{2}\right) / 2$, and by adding and subtracting these two contributions we obtain the required combinations $\hat{x}_{1}+\hat{x}_{2}$ and $\hat{p}_{1}-\hat{p}_{2}$. The spectral densities of these measurements are shown in columns (b) and (d) of Fig. 2.

The upper traces in Fig. 2 correspond to the coherent amplitudes of the input states and of the joint estimates, whereas the lower traces are the powers associated with the noise levels, all of which are at the shot noise level. The signal-to-noise ratio of the estimate is clearly larger than that of the prepared states; the coherent amplitudes of the amplitude and phase quadratures are increased by 3.0 and $2.9 \mathrm{~dB}$, respectively, which effectively correspond to noise equivalent power of $\Delta_{x}=0.51 \pm 0.02$ and $\Delta_{p}=0.52 \pm$ 0.02 shot noise units. Using Eq. (11), the fidelity is calculated to be $F^{*}=79.5 \pm 0.7 \%$, thus clearly surpassing the classical local fidelity of $2 / 3$ and close to the theoretical value $4 / 5$.

In summary, we have predicted the existence of a quantum effect exhibited by classically correlated quasiclassical states. This has been experimentally tested using a pair of sideband-encoded coherent states produced by modulating a continuous-wave laser beam. We have unfortunately not been able to prove the optimality of the joint measurement of $|\alpha\rangle\left|\alpha^{*}\right\rangle$ giving $F^{*}$ because the technique we used to prove the optimality of $F$ happened to be hard to adapt. However, we conjecture that it is the case; this is a topic for further investigation.

We thank Gerd Leuchs for useful discussions, and acknowledge financial support from the EU under project COVAQIAL (No. FP6-511004). A. A. and M. N. thank the Spanish MEC, under a "Ramón y Cajal" grant, and Fundación Ramón Areces for financial support. R. G.-P. and J.N. acknowledge support from the Belgian foundation FRIA.

[1] A. Peres and W. K. Wootters, Phys. Rev. Lett. 66, 1119 (1991).

[2] S. Massar and S. Popescu, Phys. Rev. Lett. 74, 1259 (1995).

[3] N. Gisin and S. Popescu, Phys. Rev. Lett. 83, 432 (1999).

[4] C. H. Bennett et al., Phys. Rev. A 59, 1070 (1999).

[5] G. J. Pryde et al., Phys. Rev. Lett. 94, 220406 (2005).

[6] E. R. Jeffrey et al., Phys. Rev. Lett. 96, 150503 (2006).

[7] N. J. Cerf and S. Iblisdir, Phys. Rev. A 64, 032307 (2001).

[8] S. Stenholm, Ann. Phys. (N.Y.) 218, 233 (1992).

[9] K. Hammerer, M. M. Wolf, E. S. Polzik, and J. I. Cirac, Phys. Rev. Lett. 94, 150503 (2005).

[10] V. Giovannetti et al., Phys. Rev. A 70, 022328 (2004).

[11] S. L. Braunstein, C. A. Fuchs, and H. J. Kimble, J. Mod. Opt. 47, 267 (2000).

[12] U. L. Andersen, V. Josse, and G. Leuchs, Phys. Rev. Lett. 94, 240503 (2005). 